Research Article

\title{
The Modelling of Digital Twins Technology in the Construction Process of Prefabricated Buildings
}

\author{
Yun Zhou iD, Xiao Wei, and Ying Peng \\ College of Architecture and Urban Planning, Chongqing Jiaotong University, Chongqing, China \\ Correspondence should be addressed to Yun Zhou; 990020803008@cqjtu.edu.cn
}

Received 8 October 2021; Accepted 10 December 2021; Published 30 December 2021

Academic Editor: Zhihan Lv

Copyright (c) 2021 Yun Zhou et al. This is an open access article distributed under the Creative Commons Attribution License, which permits unrestricted use, distribution, and reproduction in any medium, provided the original work is properly cited.

In the process of building construction, traditional architectural design and construction methods take a long time. The built buildings perform poorly in terms of energy usage and energy conservation. The study expects to explore the potential safety hazards of prefabricated buildings during the construction process. On this basis, a modelling study of the construction process is carried out. The study uses Digital Twins (DTs) technology and prefabricated Building Information Modelling (BIM) to conduct in-depth modelling research on the building construction process. The prefabricated building construction system oriented to DTs technology can well solve the problems of structural damage and deformation in the production, transportation, and assembly process of building components. Especially in prefabricated buildings, it can monitor and accurately predict the damage of building components that may occur in the entire system due to structural problems and material problems in real time. Regarding the building information model, the study uses third-party software to transfer the assembly information to the network cloud to further realize the display of the BIM. The study shows that the maximum value of the effective risk cases selected is 130 , and its effective rate is $100 \%$; after processing the data, it is found that the initial value is always stable, and its value is 1 ; the extracted value is always changing, the maximum value is 0.86 , and the minimum is 0.75 . By this result, the conclusion is that DTs technology and BIM can effectively monitor the indicators of risk problems during the construction of prefabricated buildings and can further reduce potential safety hazards. Through building information modelling, the development of intelligent industrialization of building construction design and the in-depth study of construction modelling has practical application value.

\section{Introduction}

With the vigorous development of science and technology, society is advancing rapidly, and the country's economic development is getting faster and faster. These changes and developments are inseparable from the contributions made by the construction industry [1-3]. The construction field has gradually changed from the traditional building of houses to the mode of intelligent and scientific construction. With the acceleration of the process of urbanization in China, the construction of prefabricated buildings [4-6] has gradually become a new trend of the times. Under this new trend, the system design and modelling of prefabricated buildings [7-9] have become the focus of attention by related researchers in the architectural field.
The in-depth analysis is conducted from a broader perspective. By the traditional architectural system, prefabricated buildings [10-12] optimize the allocation and combination of resources for the structure, system, and management of traditional buildings according to the needs of customers. Prefabricated buildings enable customers to further experience more efficient, comfortable, and acceptable humanized building clusters. The biggest difference between prefabricated buildings and traditional buildings is that prefabricated buildings are essentially industrialized manufacturing. It can customize all the construction of the building according to the customer's predetermined order and transport the new construction to the job site for reasonable assembly. The advantage of prefabricated buildings is that they can save a lot of time and complete tasks with high quality. The disadvantage of prefabricated buildings is 
that the cost is too high and the height of the building is limited. Relevant researchers have conducted a lot of research on the construction of prefabricated buildings. Studies have shown that the rapid development of prefabricated buildings in Denmark is very prominent. Meanwhile, researchers put forward the legalization of modulus and successfully used it as a standard blueprint for modulus coordination. For the building assembly system, this overcomes the original assembly monotonous architectural style and realizes the diversification of the building construction process. The development of prefabricated buildings in China is to gain experience from Soviet architecture and develop in-depth. In recent years, Digital Twins (DTs) technology [13-15], as a concept beyond reality, has been widely used in product design, product manufacturing, medical analysis, and engineering construction. The DTs technology can map the building entities in the real physical space to the virtual space, which has a certain impact on the construction of prefabricated buildings. DTs technology [16-18] is applied to prefabricated buildings, enabling the entire system to realize intelligent engineering from a single building component, a single device to a large-scale building. On the construction and safety issues in the building process, $\mathrm{Li}$ et al. [19] used solar-driven photoelectrochemical technology to study building materials. The results show that low-viscosity dopants can increase the construction strength of buildings. Shams et al. [20] studied the safety construction restoration planning in building renovation projects. They have combined the cost and duration of the project to evaluate alternative construction schedules. The results show that the construction of fire safety facilities can have an impact on the final construction schedule. Kochovski et al. [21] used the fog computing method to construct smart and safe building applications. The privacy protection data management through the smart contract of Ethereum shows that the use of fog computing helps to improve the response rate, privacy, and security.

According to the existing research and the problems, to make the prefabricated building more efficient in the construction process, the study adopts the DTs technology and the Building Information Modelling (BIM) to discuss the process modelling of prefabricated building. The innovation here is that the construction process in the real physical environment is easily affected by factors such as weather conditions and air pollution. The DTs technology is introduced to map the actual situation of the prefabricated building to the virtual space and to conduct further modelling research. Combining BIM and DTs technology, prefabricated building construction has been studied. The prefabricated building is provided with theoretical support, which is of great significance.

\section{Materials and Methods}

2.1. DTs Technology. The prefabricated building construction system of DTs technology [22-24] can solve the problems of structural damage and deformation in the production, transportation, and assembly process of building components. It should be noted that the DTs technology in prefabricated buildings can monitor and accurately predict the damage of building components in the entire system, which may occur due to structural problems and material problems. The realization of this process can effectively improve the safety and reliability of the prefabricated building in the entire construction process. In addition, DTs technology [25-27] can also effectively improve the construction process of prefabricated buildings. Figure 1 is a framework diagram of the DTs application.

DTs technology is the digital representation of physical processes, people, places, systems, or equipment. DTs technology can promote the development of industry towards intelligent development, and it is an important guarantee for the development and construction of modern cities. Many scientific research scholars have conducted research on the digitalization process of intelligent industry. In response to the challenges faced by traditional manufacturing, some scholars have proposed a DTs-driven sustainability evaluation information system structure for intelligent manufacturing and dynamic evolution throughout the life cycle. In the end, the effectiveness of the proposed DTs information architecture and sustainability evaluation method is verified. Related researchers have constructed a virtual intelligent network space architecture Intelligent DTs-Software Defined Virtual Networks (IDT-SDVNs). Simulation and performance analysis of IDT-SDVNs have verified its effectiveness. Others have used machine learning and DTs technology to build an ECG heart rhythm classifier to diagnose and detect heart diseases. Experts in related fields have found that the combination of accurate BIM and big data generated by Internet of Things (IoT) sensors makes the data in modern cities more accurate and transparent. The public and open DTs model makes smart city industrialization planning more precise. The meaning of DTs technology is to simulate the real objects in real life through digital models in the real world, to construct virtual models of different dimensions and different disciplines. The characteristics of this virtual model are the combination of virtual and real, iterative operation, and realtime interaction. Prefabricated buildings can fully apply this technology. Figure 2 is a framework of the interaction between the virtual space and the real space of DTs.

DTs technology is widely used. Due to its technical characteristics, the difficulty of integrating intelligent manufacturing and physical space, and the key characteristics in practice, it is used by various industries. The management and control system for DTs technology includes three parts: the prefabricated building application layer, the prefabricated building model layer, and the prefabricated building. Each part is divided into different subsystems. The subsystems of the application layer are collaborative process planning, optimization of production parameters, and management and control of the production environment. The subsystems of the model layer are the design and manufacturing collaboration model, the production management optimization model, and the quality control management model. The subsystems of the data layer are environmental data, material data, and machine data. Figure 3 presents the operation and maintenance of the prefabricated building system oriented to DTs.

Prefabricated buildings are complicated in the process of component transportation and assembly, and knowledge of 


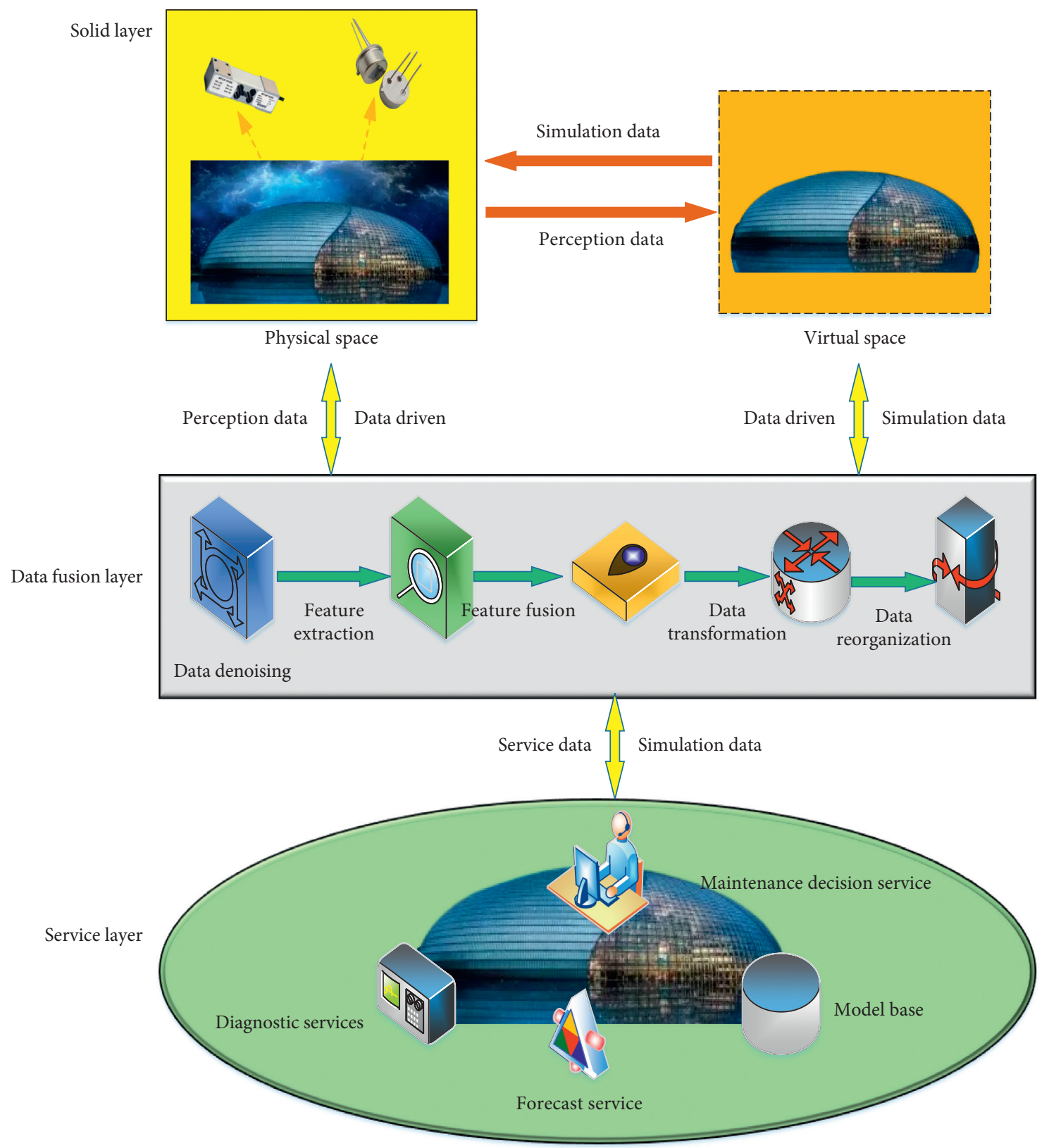

Figure 1: DTs application framework.

many disciplines has been applied. The management control system is designed and constructed, and the construction of this system has brought great benefits. The management and control system oriented to DTs can carry out good scheduling control in all aspects of building structure and materials. Figure 4 is a frame structure diagram of DTs and the production process.

The measurement and control of prefabricated building entities is an important communication method between DTs technology and building entities. The data collection of the prefabricated building is completed through monitoring sensors, which further drives DTs. In this process, the displacement and rotation torque of the building components during the assembly process will be effectively monitored. The data are transmitted and processed, and each element of the assembly process is effectively controlled. Figure 5 is the solid frame of the survey and assembly building.

In the model algorithm, the collected prefabricated building image data are expressed as the following objective function. The principle of the objective function can be expressed as follows:

$$
\min _{w, b, s, y_{u}} \frac{1}{2} w^{T} w+\lambda_{1} \sum_{i=1}^{n} u_{i i}\left(1-\left(w^{T} x^{i}+b\right) y_{i}\right) .
$$

In Equation (1), $w$ represents the weight matrix, $w^{T}$ represents the transposition of the weight matrix, $x^{i}$ represents the input, $y_{i}$ represents the output, $b$ represents the deviation, $\lambda_{1}$ represents the eigenvalue of the matrix, and $u_{i i}$ represents the element value of the $i$-th row and the $\mathrm{i}$-column. 


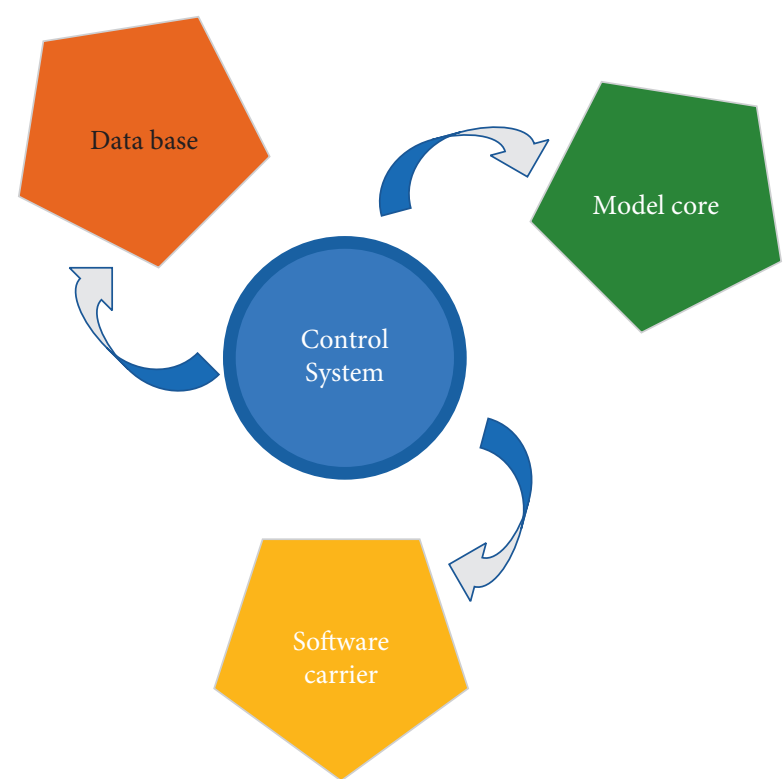

FIGURE 2: A framework of the interaction between the virtual space and the real space of DTs.

$$
\text { s.t. } \forall i, s_{i} 1 \geq 0, F \in R^{n \times c}, F^{T} F=I .
$$

In Equation (2), $s$ is the similarity matrix between samples. Equation (2) is a supplement to Equation (1).

$$
L_{s}=D_{s}-\frac{s^{T}+s}{2} \text {. }
$$

In Equation (3), $D_{s} \in R^{n \times n}$ refers to a diagonal element, $s$ represents the coefficient matrix and $s^{T}$ represents the transposition of the coefficient matrix.

$$
\sum_{j} \frac{\left(s_{i j}+s_{j i}\right)}{2} \leftarrow y_{u}^{(t)} t=t+1 \text {. }
$$

When further training the constructed model algorithm, the learning rate update strategy and the polynomial decay learning rate adjustment method are adopted, specifically expressed as follows:

$$
\text { init }_{l} r \times\left(1-\frac{\text { epoch }}{\text { max_epoch }}\right)^{\text {power }} .
$$

The initial learning rate is 0.0004 , and the power is set to 0.8 . In the training process, weighted cross entropy (WCE) is used as a cost function to optimize model training. $z_{k}(x, \theta)$ refers to the unnormalized log probability value of the pixel $x$ of the k-th category under the given network parameters. The definition of softmax function $p_{k}(x, \theta)$ is shown as follows:

$$
p_{k}(x, \theta)=\frac{\exp \left\{z_{k}(x, \theta)\right\}}{\sum_{k}^{K} \exp \left\{z_{k_{\prime}}(x, \theta)\right\}} .
$$

In Equation (6), $K$ represents the total number of image data categories. In the prediction phase, when Equation (6) takes the maximum, pixel $x$ is marked as the k-th category; $k *=\arg \max \left\{P_{k}(x, \theta)\right\}$. For ease of description, note $N$ as the total number of pixels contained in the training batch image data. $y_{i}$ means the true semantic annotation of pixel $x_{i} \cdot p_{k}\left(x_{i}, \theta\right)$ represents the prediction probability that pixel $x_{i}$ belongs to the k-th semantic category. In other words, the probability value after logarithmic normalization is simply marked as $p_{i k}$. The training process aims to find the optimal network parameter $\theta *$ by minimizing the WCE loss function $\ell(x, \theta)$; that is, $\theta *$ is $\theta *=\min \ell(x, \theta)$. In the process of assembly building construction, the unbalanced training samples in the extracted image data usually make the network focus on some easily separable categories. In order to solve the problem of poor sample identification, Online Hard Example Mining (OHEM) strategy is used in the experiment to optimize the network training process. The improved loss function is shown as follows:

$\ell(x, \theta)=-\frac{1}{\sum_{i=1}^{N} \sum_{k=1}^{K} \delta\left(y_{i}=k, p_{i k}<\eta\right)} \sum_{i=1}^{N} \sum_{k=1}^{K} \delta\left(y_{i}=k, p_{i k}<\eta\right) \log p_{i k}$,

$\eta \in(0,1]$ means a predefined threshold, and $\delta(\cdot)$ means a symbolic function. When the condition satisfies, it is equal to 1 ; otherwise, it is 0 . The definition of loss function of assembly building construction fusion weighting is shown as follows:

$$
\ell(x, \theta)=-\sum_{i=1}^{N} \sum_{k=1}^{K} w_{i k} q_{i k} \log p_{i k}
$$

$q_{i k}=q\left(y_{i}=k \mid x_{i}\right)$ refers to the true label distribution of pixel $x_{i}$ in the k-th category. $w_{i k}$ is the weighted coefficient. In the training process, the calculation strategy in Equation (9) is adopted:

$$
w_{i k}=\frac{1}{\ln \left(c+p_{i k}\right)}
$$

In Equation (9), $c$ refers to the extra super parameter, which is set to 1.0 during the experiment.

2.2. Prefabricated BIM. In the prefabricated building system, if the building is compared with a physical system, then the intelligent building is the effective integration of people, building entities, and building information virtual entities. The life cycle (LC) of prefabricated buildings is to use artificial intelligence, big data, and IoT to predict. When some scholars evaluated the LC of prefabricated buildings, they proposed a link prediction method by similarity, which only relies on limited known data to estimate the missing unit process data. This calculation method shows the potential of LC prediction and estimation in prefabricated buildings. Some foreign experts proposed an energy management system model of fabricated building microgrid by battery energy storage. The simulation suggests that the framework can estimate the construction operation cost more accurately and improve the overall performance of the battery as a flexible resource in the construction microgrid. In addition, some people have proposed a two-stage optimization model framework for the integrated optimization planning of renewable energy resources (RERs) 


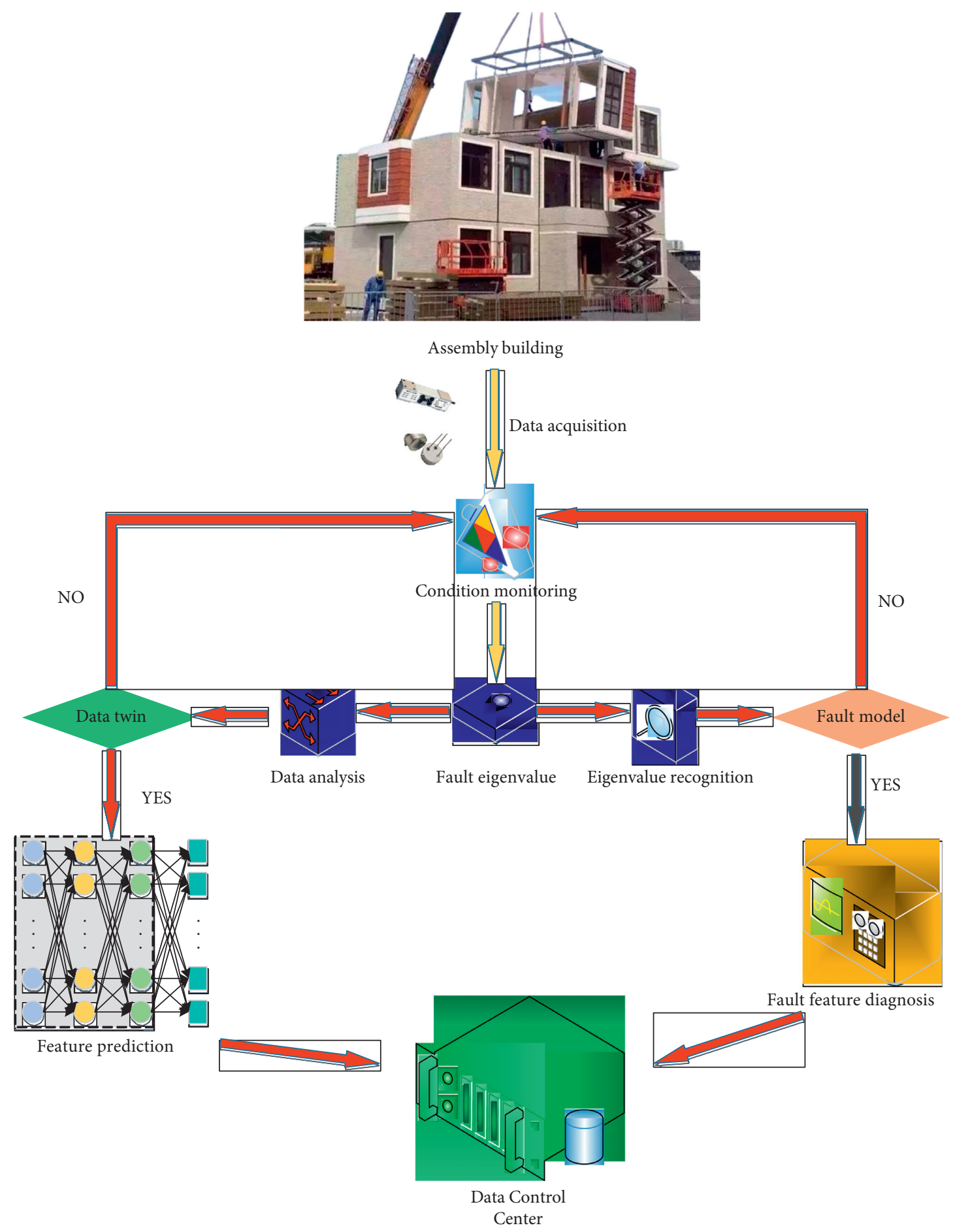

FIGURE 3: Operation and maintenance of the prefabricated building system for DTs.

and batteries in prefabricated buildings by prosumer energy management. Finally, the effectiveness is verified by performance evaluation. In the field of prefabricated buildings, some scholars have predicted the LC of power transformers. It is found that the mean absolute percentage error (MAPE) of the model on the test set is only $5.20 \%$, and the accuracy is significantly higher. Although there are many data analysis studies on prefabricated buildings in the urban process, there are few studies on the analysis of prefabricated buildings in smart cities by using DTs technology, and there are few studies on the digital development of prefabricated buildings. A risk prediction model by DTs technology is proposed for the feature extraction of multisource data of prefabricated buildings, which is of great significance to the digital development and safety maintenance of prefabricated buildings in modern cities.

BIM [28-30] can be displayed in the form of web pages. By its complexity, the study uses third-party software to transmit assembly information to the network cloud to 


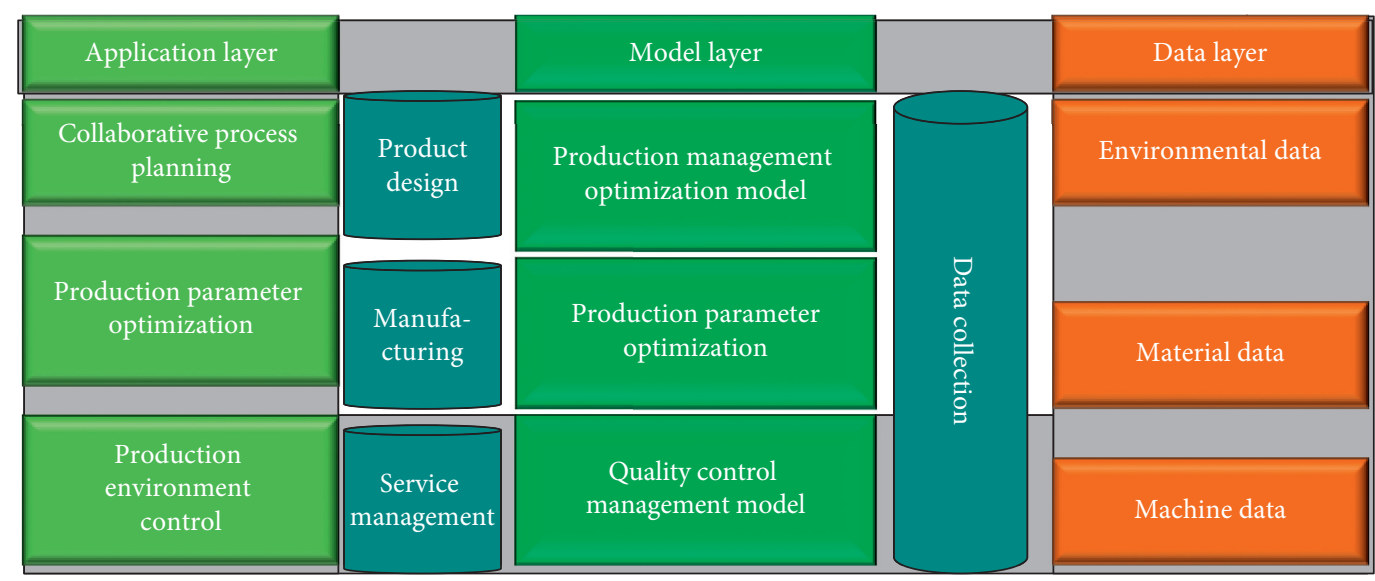

Figure 4: Frame structure of DTs and production process.

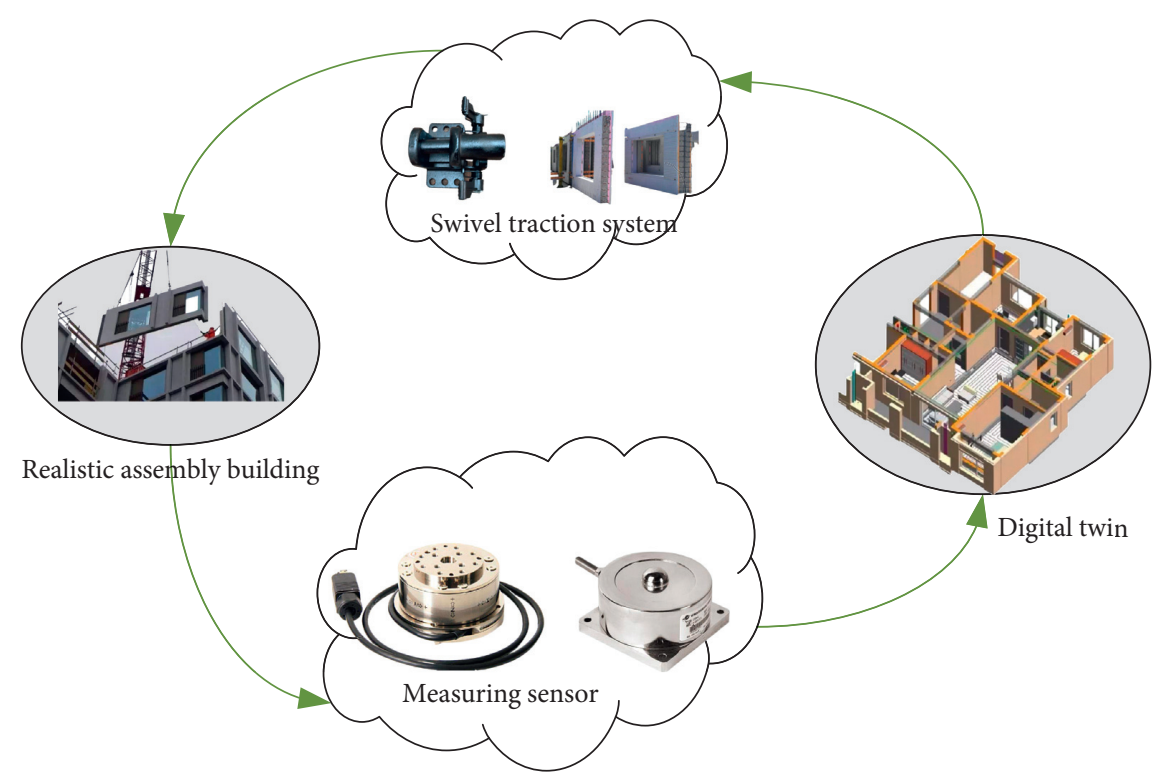

Figure 5: Measured and assembled building entities.

further realize the BIM model presentation. In the BIM platform, the study can zoom in and zoom out the BIM model. Figure 6 shows the BIM model of assembly building rotating cloud.

The assembly BIM can conduct in-depth and effective modelling research on the construction process. In addition, the prefabricated construction system for DTs technology can well solve the structural damage and deformation of construction components in the production, transportation, and assembly process. It can real-time monitor and accurately predict the damage of building components due to structural and material problems in the whole system. The practical application of the BIM model in China can be reflected in the four stages of design, assembly, maintenance, and operation management. Figure 7 is the process diagram of BIM technology application.

The monitoring data of the assembly construction process are simulated and analysed, and the whole construction process is effectively monitored by feedback control. The construction monitoring process of the building is shown in Figure 8. First, the drawings are designed, and the specific construction scheme is formulated into equations. Second, the design compliance calculation and presimulation calculation are carried out. Third, the monitoring instruction file is issued for the next stage of construction, and further construction monitoring is carried out. Finally, data are analysed. Figure 8 is the schematic diagram of the assembly building construction monitoring process.

\section{Results}

3.1. Analysis of Construction Results of Prefabricated Buildings Oriented to DTs Technology. The study models according to the DTs technology prefabricated building. Regarding the risk in construction, data processing of specific indicators is carried out, and the specific results are shown in Figure 9. Cronbach's alpha is used to measure the reliability of the 


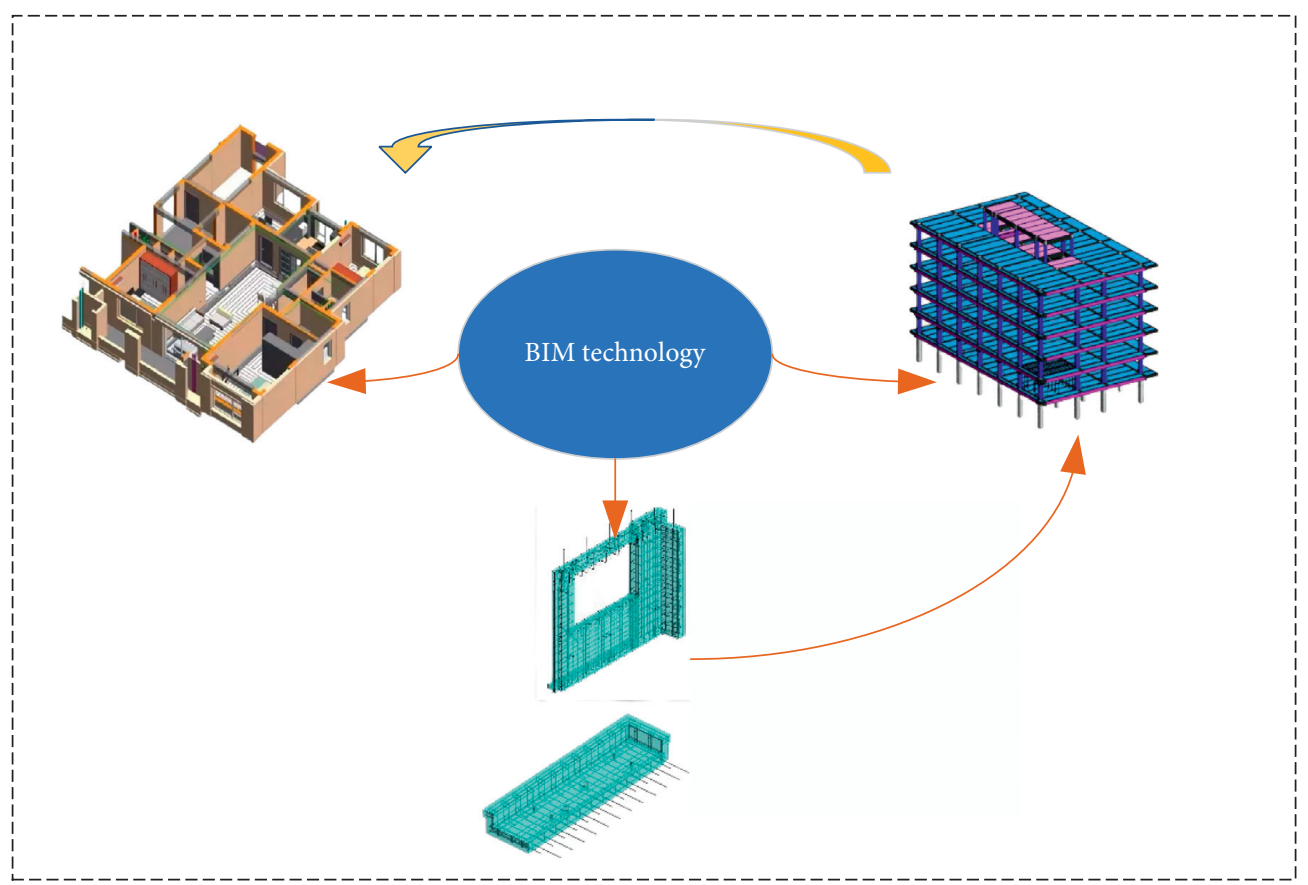

FIGURE 6: BIM model of assembly building turning cloud.

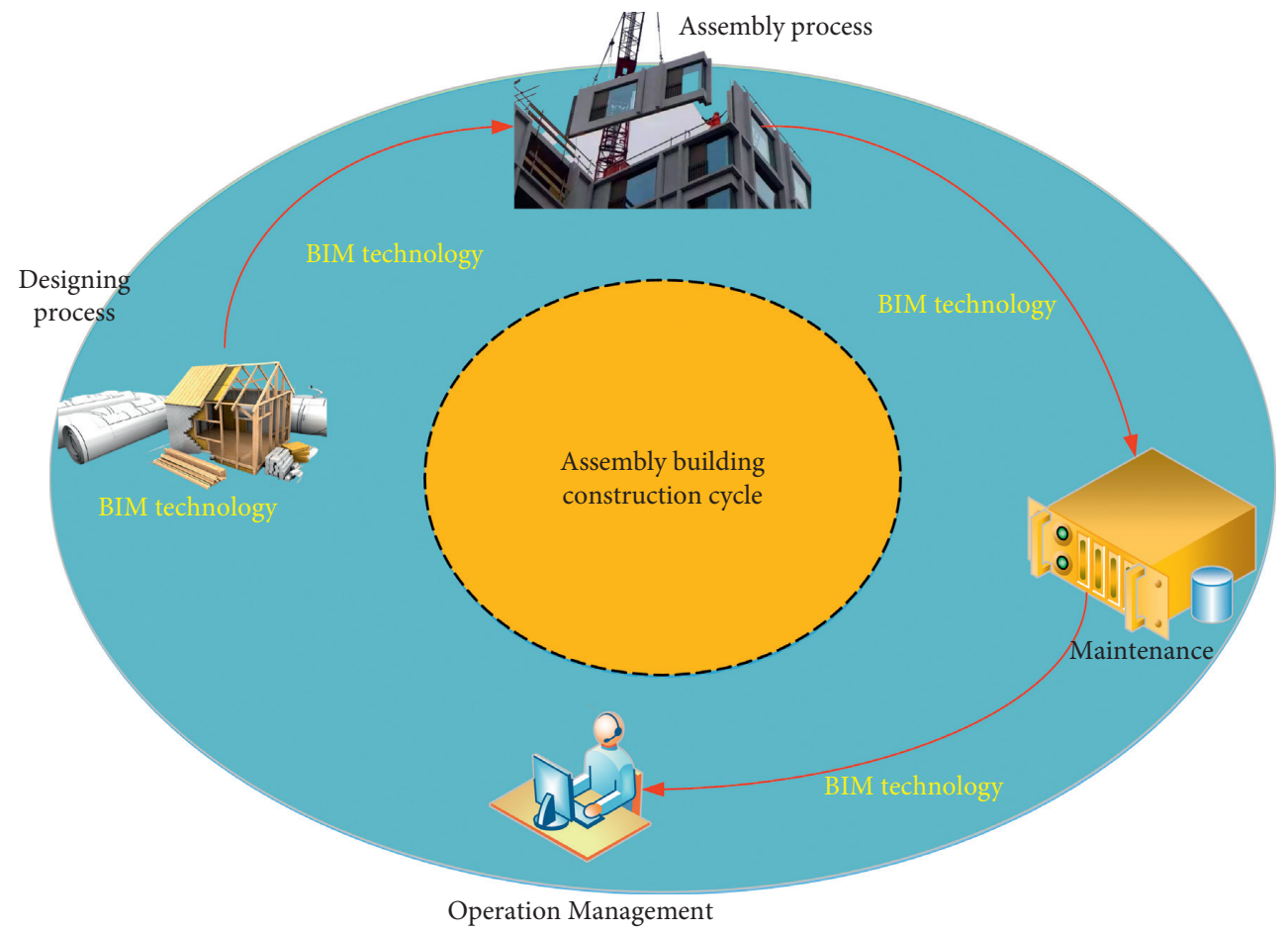

FIGURE 7: Diagram of BIM technology application process.

data. Cronbach's alpha value is between 0 and 1 , and the value in the $0.8-0.9$ interval is considered to be very reliable. For valid risk cases, when the maximum value is 130 , the effective rate is $100 \%$. For invalid cases, its value is 0 , which means that the collected data are all valid. Figure 9 is a data diagram of the risk index situation of the prefabricated building construction.
Figure 9 shows the common factor variance data of the risk indicators during the construction of the prefabricated building, so the Kaiser-Meyer-Olkin (KMO) factor needs to be verified. In general, the value of $\mathrm{KMO}$ is greater than 0 and less than 1 . The closer the KMO value to 1 , the stronger the correlation between the factors. If the value of KMO is closer to 0 , the correlation between the factors is poor. If the 


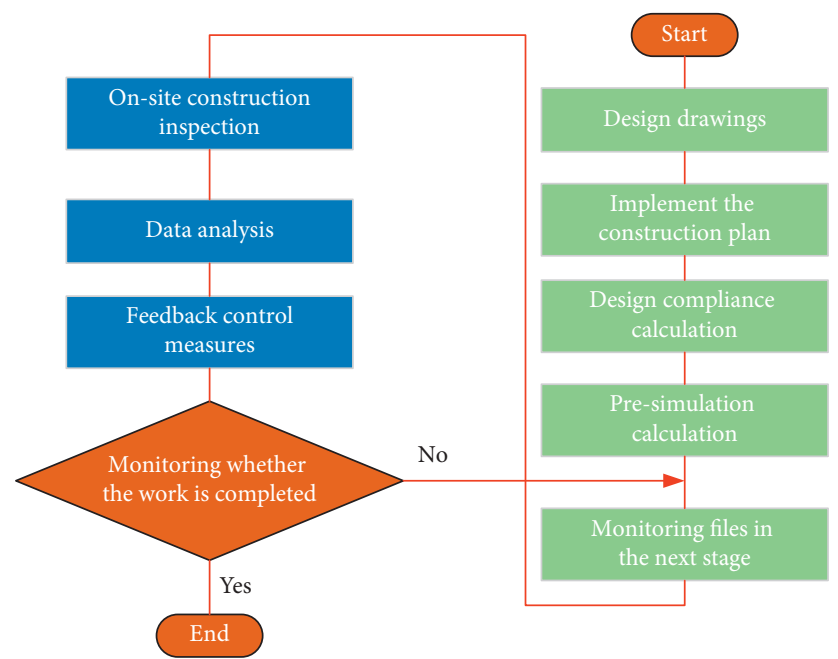

FIGURE 8: Schematic diagram of the monitoring process of prefabricated building construction.

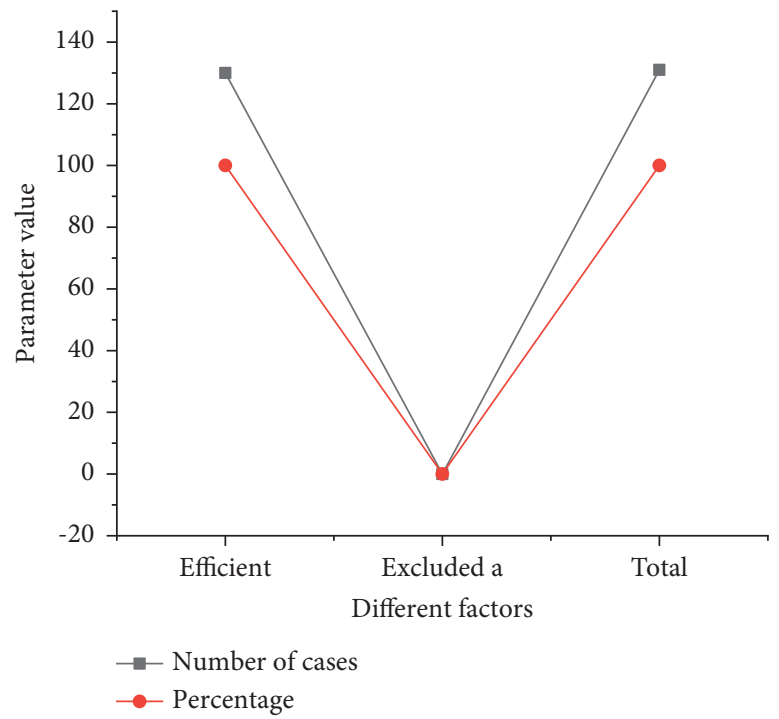

Figure 9: Data diagram of risk indicators for prefabricated building construction.

value of KMO is less than 0.5 , the selected index is not suitable for factor analysis. After processing the data, it is found here that the initial value has been very stable, and its value is 1 . The extracted value is always changing, the maximum is 0.86 , and the minimum is 0.75 . The experimental results show that the correlation between the factors is better. Figure 10 is a data diagram of the variance of the common factor of the risk indicators of the prefabricated building during the construction process.

3.2. Analysis of BIM Results. The research analyses the data through BIM, combined with the specific conditions of the risk indicators, and the results shown in Figure 11 are obtained. In Figure 11(a), the maximum value of variance is $52.12 \%$ and the maximum cumulative value of each indicator is $65.02 \%$. In Figure 11(b), the maximum value of variance is $52.12 \%$ and the maximum cumulative value of each indicator is $58.97 \%$. In Figure 11(c), the maximum value of variance is $18.45 \%$, and the maximum cumulative value of each indicator is $45.02 \%$. The influence of the indicators on the total variance is analysed and extracted, and the cumulative explanatory rate of these three indicators is $65.02 \%$. Figure 11 shows the total variance data of the risk indicators during the construction process. 


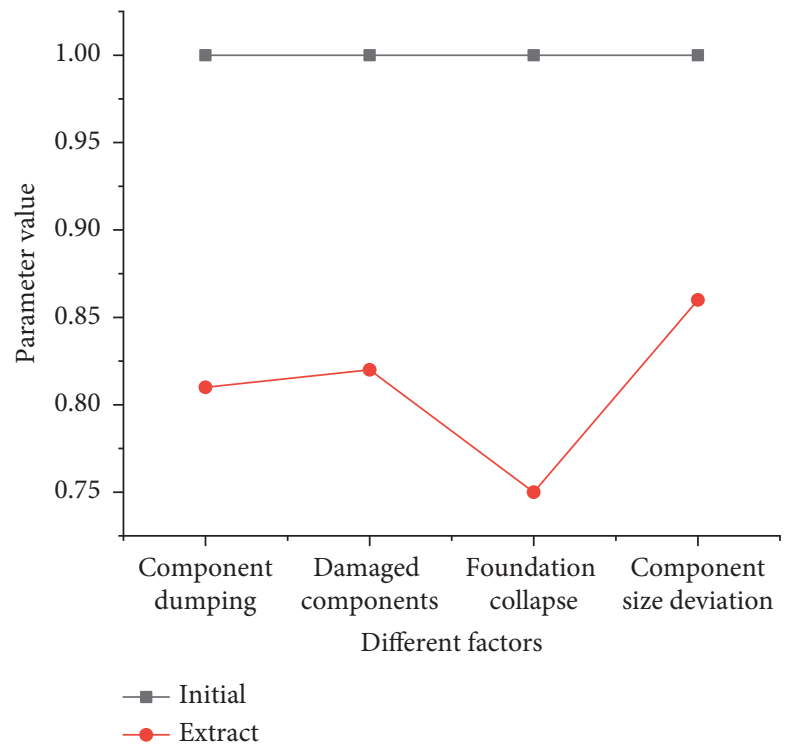

FIgURE 10: Data diagram of common factor variance of risk index of prefabricated building construction.

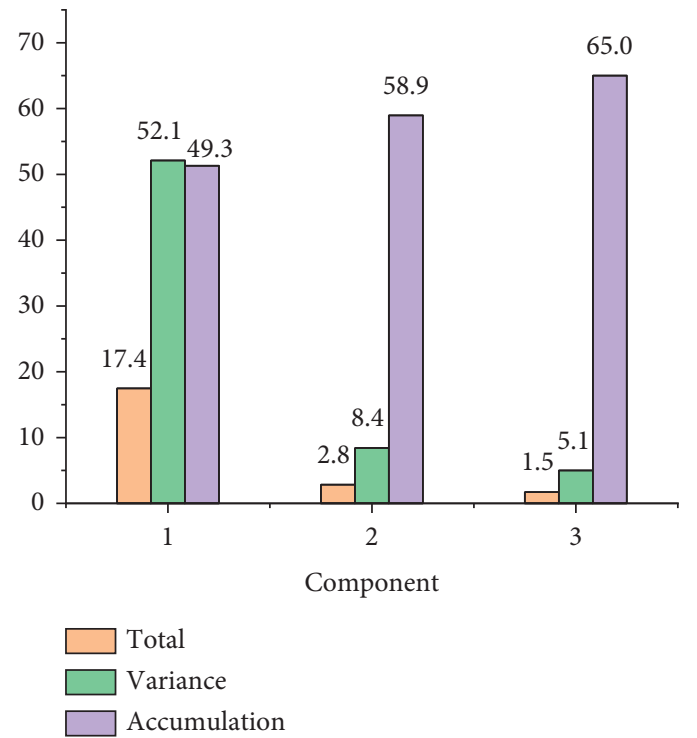

(a)

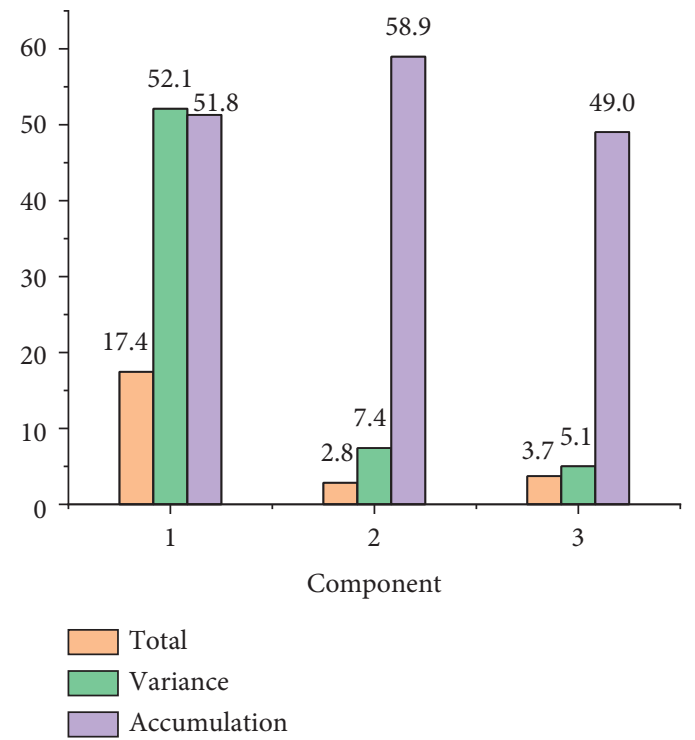

(b)

Figure 11: Continued. 


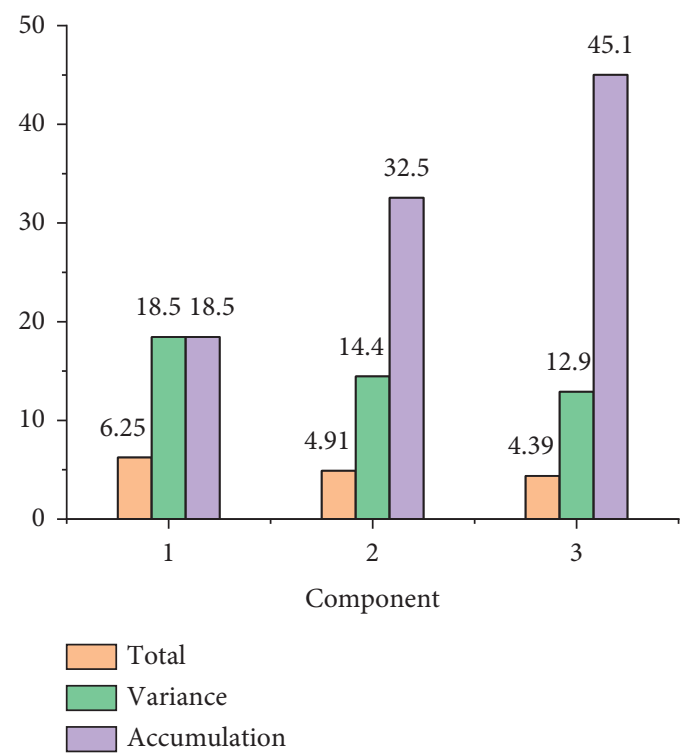

(c)

Figure 11: The total variance data of the construction risk index situation are as follows: (a) the initial feature value; (b) the extraction of the sum of squares and loaded; (c) the rotation of the sum of squares and loaded.

\section{Conclusions}

With the acceleration of China's urbanization process, prefabricated building construction has gradually become a new trend of the times. Under the trend of this new era, the system design and modelling of prefabricated buildings have become the focus. DTs technology and prefabricated BIM are used to conduct in-depth modelling research on the building construction process. The method of building an information model is adopted, and third-party software is used to transmit the assembly information to the network cloud. Through the in-depth modelling research of the building construction process, the BIM display is further realized. The prefabricated building construction system oriented to DTs technology can well solve the problems of structural damage and deformation that occur during the production, transportation, and assembly of building components. Prefabricated buildings can monitor and accurately predict the damage of building components that may occur in the entire system in real time. This study uses third-party software to transfer the assembly BIM to the network cloud to further realize the BIM model display. The conclusion is as follows: DTs technology and BIM effectively monitor the indicators of risk problems in the construction process of prefabricated buildings. Monitoring can effectively guide the designer on the potential safety hazards that may arise when building components are assembled. The disadvantage here is that the index selection in the construction process is not comprehensive enough, and the data collection area is narrow. These deficiencies have a certain impact on the universality of research. In the future, multilevel data will be selected, research data will be supplemented, and the deviation of research results will be reduced.

\section{Data Availability}

The data used to support the findings of this study are available from the corresponding author upon request.

\section{Conflicts of Interest}

The authors declare that there are no conflicts of interest.

\section{References}

[1] S. Navaratnam, T. Ngo, T. Gunawardena, and D. Henderson, "Performance review of prefabricated building systems and future research in Australia," Buildings, vol. 9, no. 2, p. 38, 2019.

[2] L. Luo, G. Qiping Shen, G. Xu, Y. Liu, and Y. Wang, "Stakeholder-associated supply chain risks and their interactions in a prefabricated building project in Hong Kong," Journal of Management in Engineering, vol. 35, no. 2, Article ID 05018015, 2019.

[3] X. Li, C. Wang, A. Alashwal, and S. Bora, "Game analysis on prefabricated building evolution based on dynamic revenue risks in China," Journal of Cleaner Production, vol. 267, Article ID 121730, 2020.

[4] Y. Wang, X. Xue, T. Yu, and Y. Wang, "Mapping the dynamics of China's prefabricated building policies from 1956 to 2019: a bibliometric analysis," Building Research \& Information, vol. 49, no. 2, pp. 216-233, 2021.

[5] L. Luo, X. Liang, C. Fang, Z. Wu, X. Wang, and Y. Wang, "How to promote prefabricated building projects through internet of things? A game theory-based analysis," Journal of Cleaner Production, vol. 276, Article ID 124325, 2020.

[6] N. Sebaibi and M. Boutouil, "Reducing energy consumption of prefabricated building elements and lowering the environmental impact of concrete," Engineering Structures, vol. 213, Article ID 110594, 2020. 
[7] X.-J. Li, "Research on investment risk influence factors of prefabricated building projects," Journal of Civil Engineering and Management, vol. 26, no. 7, pp. 599-613, 2020.

[8] J. Liu, E. Gong, D. Wang, and Y. Teng, "Cloud model-based safety performance evaluation of prefabricated building project in China," Wireless Personal Communications, vol. 102, no. 4, pp. 3021-3039, 2018.

[9] K. M. A. El-Abidi, G. Ofori, S. A. S. Zakaria, and A. R. A. Aziz, "Using prefabricated building to address housing needs in Libya: a study based on local expert perspectives," Arabian Journal for Science and Engineering, vol. 44, no. 10, pp. 8289-8304, 2019.

[10] A. Alkhalidi, A. Abuothman, A. AlDweik, and A.-H. AlBazaz, "Is it a possibility to achieve energy plus prefabricated building worldwide?" International Journal of Low Carbon Technologies, vol. 16, no. 1, pp. 220-228, 2021.

[11] Z. Yuan, G. Ni, L. Wang et al., "Research on the barrier analysis and strength measurement of a prefabricated building design," Sustainability, vol. 12, no. 7, p. 2994, 2020.

[12] M. Ma, K. Zhang, L. Chen, and S. Tang, "Analysis of the impact of a novel cool roof on cooling performance for a lowrise prefabricated building in China," Building Service Engineering Research and Technology, vol. 42, no. 1, pp. 26-44, 2021.

[13] G. Bhatti, H. Mohan, and R. Raja Singh, "Towards the future of smart electric vehicles: digital twin technology," Renewable and Sustainable Energy Reviews, vol. 141, Article ID 110801, 2021.

[14] R. He, G. Chen, C. Dong, S. Sun, and X. Shen, "Data-driven digital twin technology for optimized control in process systems," ISA Transactions, vol. 95, pp. 221-234, 2019.

[15] K. J. Wang, T. L. Lee, and Y. Hsu, "Revolution on digital twin technology-a patent research approach," International Journal of Advanced Manufacturing Technology, vol. 107, no. 11, pp. 4687-4704, 2020.

[16] R. Anderl, S. Haag, K. Schützer, and E. Zancul, "Digital twin technology - an approach for Industrie 4.0 vertical and horizontal lifecycle integration," It - Information Technology, vol. 60, no. 3, pp. 125-132, 2018.

[17] B. R. Barricelli, E. Casiraghi, and D. Fogli, "A survey on digital twin: definitions, characteristics, applications, and design implications," IEEE access, vol. 7, pp. 167653-167671, 2019.

[18] J. Ma, H. Chen, Y. Zhang, and H. Guo, "A digital twin-driven production management system for production workshop," International Journal of Advanced Manufacturing Technology, vol. 110, no. 5, pp. 1385-1397, 2020.

[19] H. Li, B. Liu, S. Feng, H. Li, T. Wang, and J. Gong, "Construction of uniform buried pn junctions on pyramid $\mathrm{Si}$ photocathodes using a facile and safe spin-on method for photoelectrochemical water splitting," Journal of Materials Chemistry, vol. 8, no. 1, pp. 224-230, 2020.

[20] S. T. Shams Abadi, N. Moniri Tokmehdash, A. Hosny, and M. Nik-Bakht, "BIM-based co-simulation of fire and occupants' behavior for safe construction rehabilitation planning," Fire, vol. 4, no. 4, p. 67, 2021.

[21] P. Kochovski and V. Stankovski, "Building applications for smart and safe construction with the DECENTER fog computing and brokerage platform," Automation in Construction, vol. 124, Article ID 103562, 2021.

[22] A. Liljaniemi and H. Paavilainen, "Using digital twin technology in engineering education - course concept to explore benefits and barriers," Open Engineering, vol. 10, no. 1, pp. 377-385, 2020.
[23] G. P. Agnusdei, V. Elia, and M. G. Gnoni, "Is digital twin technology supporting safety management? a bibliometric and systematic review," Applied Sciences, vol. 11, no. 6, p. $2767,2021$.

[24] M. Liu, S. Fang, H. Dong, and C. Xu, "Review of digital twin about concepts, technologies, and industrial applications," Journal of Manufacturing Systems, vol. 58, pp. 346-361, 2021.

[25] C. Zhuang, T. Miao, J. Liu, and H. Xiong, "The connotation of digital twin, and the construction and application method of shop-floor digital twin," Robotics and Computer-Integrated Manufacturing, vol. 68, Article ID 102075, 2021.

[26] Q. Liu, J. Leng, D. Yan et al., "Digital twin-based designing of the configuration, motion, control, and optimization model of a flow-type smart manufacturing system," Journal of Manufacturing Systems, vol. 58, pp. 52-64, 2021.

[27] T. R. Wanasinghe, L. Wroblewski, B. K. Petersen et al., "Digital twin for the oil and gas industry: overview, research trends, opportunities, and challenges," IEEE Access, vol. 8, pp. 104175-104197, 2020.

[28] A. Costin, A. Adibfar, H. Hu, and S. S. Chen, "Building Information Modeling (BIM) for transportation infrastructure - literature review, applications, challenges, and recommendations," Automation in Construction, vol. 94, pp. 257-281, 2018.

[29] S. Tang, D. R. Shelden, C. M. Eastman, P. Pishdad-Bozorgi, and X. Gao, "A review of building information modeling (BIM) and the internet of things (IoT) devices integration: present status and future trends," Automation in Construction, vol. 101, pp. 127-139, 2019.

[30] F. Rezaei, C. Bulle, and P. Lesage, "Integrating building information modeling and life cycle assessment in the early and detailed building design stages," Building and Environment, vol. 153, pp. 158-167, 2019. 